\title{
Emerging hepatotoxicity biomarkers and their potential to improve understanding and management of drug-induced liver injury
}

\author{
Jiri Aubrecht ${ }^{1 *}$, Shelli J Schomaker ${ }^{1}$ and David E Amacher ${ }^{2}$ \\ See related Research, http://genomemedicine.com/content/5/9/86
}

\begin{abstract}
Biomarkers of drug-induced liver injury (DILI) are essential for the diagnosis of severe cases of DILI in clinical trials and clinical practice, but the currently used biomarker paradigm detects damage after it has occurred and has limited prognostic value. The development of new biomarker strategies that improve the diagnosis of DILI by providing increased specificity and/or by identifying individual patients who are at risk for DILI is therefore crucial.
\end{abstract}

Drug-induced liver injury (DILI) is the leading cause of acute liver failure in the US and the most common reason for US Food and Drug Administration regulatory actions regarding approved medications. It is estimated that preclinical evaluation of drug candidates using animal studies and conventional clinical pathology measures fail to detect up to $40 \%$ of potentially hepatotoxic compounds in humans. Current biomarkers of DILI are essential for the diagnosis of severe DILI, but their ability to differentiate severe DILI cases from mild ones at the onset of DILI and/or to predict an outcome following liver injury is limited. Furthermore, there is a lack of markers capable of identifying individuals who are susceptible to DILI. Multiple groups, from individual laboratories to large-scale international collaborations, are beginning to uncover new biomarkers that might improve the DILI diagnostic paradigm. One such study, by Jürgen Borlak and colleagues, identified individuals who are susceptible to acetaminophen (APAP)-induced liver injury using a proteomics approach [1]. In common with other recent studies, the latest work implicates the immune system in DILI.

\footnotetext{
* Correspondence: Jiri.Aubrecht@pfizer.com

'Drug Safety R\&D, Pfizer Inc., Eastern Point Rd, Groton, CT, USA

Full list of author information is available at the end of the article
}

\section{Current clinical practice}

The most common circulating biomarkers used in clinical practice to detect liver injury measure either an alteration in the normal liver function (or homeostasis), such as differences in the levels of serum total bilirubin (Tbil) and total bile acids, or changes in tissue and cell integrity, which are detected by leakage of cell-specific components, such as alanine aminotransferase (ALT), aspartate aminotransferase (AST) and alkaline phosphatase (ALP), into serum. Serum elevations of ALT and AST indicate hepatocellular damage, and increased circulating ALP is linked to hepatobiliary injury. To diagnose DILI, Hy's law is regarded as the gold standard and is based on a greater than threefold elevation of serum ALT accompanied by greater than two-fold increase in Tbil in the absence of hepatobiliary injury detected using ALP. Recently, Hy's law was modified to limit 'clinically irrelevant' increases of ALT, by increasing the threshold to five-fold and including ALP to cover the hepatobiliary component of DILI (reviewed in [2]).

Although the current paradigm is useful for the diagnosis of severe DILI, there are several gaps that need to be addressed. These biomarkers detect damage after it has occurred and thus have limited prognostic value. In the case of Tbil, severe liver injury is often required before a significant increase is observed. By contrast, increases in ALT activity often indicate a transient hepatocellular injury that does not progress to severe damage (see [2] for review). Therefore, new and specific biomarkers that can complement those that are currently used and can improve the existing DILI diagnostic paradigm are essential. In particular, differentiating clinically relevant but mild cases of DILI from severe ones would improve both the diagnosis and management of DILI.

\section{Indicators of liver injury}

Both individual laboratories as well as international research projects, such as the Safer And Faster Evidence- 
based Translation (SAFE-T) consortium (started under the European Union Innovative Medicines Initiative) and the Critical Path Institute's Predictive Safety Testing Consortium, are evaluating novel biomarker approaches (for a review see [2] and Table 1 ). In a recently published study, which included the largest number of subjects to date, Schomaker and colleagues [3] evaluated the diagnostic utility of glutamate dehydrogenase (GLDH), purine nucleoside phosphorylase, malate dehydrogenase $(\mathrm{MDH})$ and paraxonase 1 for liver injury in over 800 people, including healthy people of all ages, people with a variety of liver impairments and several cases of APAP poisoning. GLDH and MDH had a correlation with elevated ALT levels and possessed a high predictive power (determined by receiver operator characteristic analysis) for liver injury. An evaluation of patients with APAPinduced liver injury provided evidence that both GLDH and MDH might have utility as biomarkers of DILI.

To address the prognostic capability of emerging biomarkers for APAP poisoning, several studies evaluated miR122, high mobility group box-1 (HMGB1), caspasecleaved keratin-18 (K18) and GLDH in patients admitted to hospital for APAP overdose [4-6]. In the first study, HMGB1 and K18 did not predict survival after APAP poisoning [6], but subsequent studies found that levels of miR122, HMGB1 and K18, which could be detected at early stages after the APAP overdose (on the admission to the hospital), provided better prognostic value than ALT and APAP blood concentrations, and were predictors of whether the subject would develop acute liver injury $[4,5]$. In addition, a recent case study demonstrated the value of these biomarkers for decisionmaking before the initiation of therapy in APAPoverdosed subjects; miR122 and K18 elevations correctly identified the patient's need for $\mathrm{N}$-acetyl cysteine therapy [7]. Although more research is needed, these findings indicate the usefulness of emerging biomarkers in the management of DILI.

\section{Personalized medicine}

The identification of individuals who are susceptible to DILI before treatment would improve drug safety. In such a paradigm, genetic or biochemical biomarkers would classify populations at risk of developing liver injury before exposure. This is the foundation of the personalized medicine approach, in which individual patients are treated with therapeutics based on their individual susceptibilities to known adverse drug effects. To date, several genome-wide association studies (GWAS) have identified specific genetic loci, such as $H L A-B * 5701, H L A-A^{*} 0201$ and HLA-DQB1*0602, that confer predisposition to liver injury from the drugs flucloxallicin, amoxicillin and clavulanate, respectively $[8,9]$. Despite these successes in identifying genetic predisposition to DILI for individual agents, the search for genetic loci that are associated with general DILI has proven to be difficult, as shown by a recent study [10]. In this GWAS of over 700 DILI patients from the USbased Drug-Induced Liver Injury Network and three international registries, a relatively weak association between STAT4 (signal transducer and activator of transcription protein 4 involved with the growth, survival and differentiation of cells and the development and function of the immune system) and DILI was observed, indicating a role for the immune system in DILI. The absence of other GWAS findings supports the idea that strong genetic determinants of DILI may be largely drug-specific or reflect rare genetic variations.

In search of non-genetic determinants of DILI susceptibility, the recently published study by Borlack and colleagues [1] used a proteomics approach to identify individuals who are susceptible to APAP-induced liver injury. In this case, healthy individuals exhibited a transient increase of ALT after treatment with the maximum allowable dose of APAP. Using differences in serum proteomic profiles (before APAP treatment) as indicators of individuals who were highly susceptible to APAP-induced liver injury, the study uncovered 20 proteins that function primarily in the acute phase and/or immune response as susceptibility factors. Both the presence of acute phase and immune response proteins before APAP treatment and the STAT4 genetic association mentioned above [10] provide further evidence for a crucial role of the immune response in predisposing individuals to DILI. More importantly, these findings provide a crucial impetus for further prospective studies to evaluate fully the ability of

Table 1 Status of circulating biomarkers of hepatotoxicity

\begin{tabular}{ccc}
\hline Type & In clinical use & Emerging \\
\hline Proteins & ALT, AST, ALP, GGT, 5'-nucleotidase, TNFa, IL-1 1, IL-6 & PON1, PNP, MDH, GLDH, ARG1, aGST, HPD, K18, HMGB1 \\
Nucleic acids & miR-122 and miR-192, mRNA Alb and mRNA Ambp \\
$\begin{array}{c}\text { Excretory function markers } \\
\text { Genetic }\end{array}$ & Bile acid profiles (conjugated vs. unconjugated) \\
& & $H L A-B^{*} 5701$, HLA-A*0201 and HLA-DQB1*0602
\end{tabular}

Alb, albumin; ALP, alkaline phosphatase; ALT, alanine aminotransferase; Ambp, a-1-microglobulin/bikunin precursor; ARG1, arginase 1; AST, aspartate aminotransferase; GGT, gamma-glutamyl transferase; GLDH, glutamate dehydrogenase; aGST, a gluthathione-S-transferase; HMGB1, high mobility group box-1 protein; HPD, 4-hydroxyphenylpyruvate dioxygenase; IL-1 $\beta$, interleukin-1 $\beta$; IL-6, interleukin-6; K18, keratin-18; MDH, malate dehydrogenase; miRNA-122, microRNA-122; miRNA-192, microRNA-192; PON1, paraoxonase 1; PNP, purine nucleoside phosphorylase; TNFa, tumor necrosis factor-a. 
genetic or biochemical biomarkers to identify populations at risk of developing liver injury.

\section{Filling the gaps}

Emerging biomarkers for human DILI show promise for improving our understanding and management of DILI cases. Nevertheless, several areas need to be addressed before these markers can be widely applied in clinical practice. Large cohorts of relevant clinical samples need to be analyzed. For ethical reasons, the development and evaluation of DILI biomarkers has mainly relied on cases of accidental APAP overdose. To increase our understanding of DILI, biomarker candidates need to be studied in both healthy volunteers and in patients with DILI caused by a wide spectrum of therapeutic agents and in those with non-drug-induced liver injury, for example in patients with liver disease or other organ injury.

Prospective clinical evaluation strategies using carefully designed clinical trials to detect drug-induced kidney, vascular and liver injury are being carried out by the SAFE-T consortium (reviewed in [2]). The complexity and feasibility of prospective clinical studies, as well as the surrounding ethical issues, require the exploration of alternative sample-collection strategies; one example would be a retrospective approach that exploits discarded samples of liver damage etiologies from patients undergoing hospital visits [3]; a second example would be the prospective collection and storage of samples from clinical trials conducted for drug development. The analysis of emerging biomarkers of hepatotoxicity in samples from large cohorts of diverse subjects will build confidence and broader acceptance of the markers, and potentially lead to improved understanding and management of DILI.

\section{Abbreviations}

ALP: alkaline phosphatase; ALT: alanine aminotransferase;

APAP: acetaminophen; AST: aspartate aminotransferase; DILI: drug-induced

liver injury; GLDH: glutamate dehydrogenase; GWAS: genome-wide association studies; HMGB1: high mobility group box-1; K18: keratin-18; MDH: malate dehydrogenase; SAFE-T: Safer And Faster Evidence-based Translation; STAT4: signal transducer and activator of transcription protein 4; Tbil: total bilirubin.
}

\section{Competing interests}

The authors declare that they have no competing interests.

\section{Author details}

${ }^{1}$ Drug Safety R\&D, Pfizer Inc., Eastern Point Rd, Groton, CT, USA. ${ }^{2}$ Toxadvisor Toxicology Consulting, Hadlyme, CT, USA.

Published: 27 September 2013

\section{References}

1. Borlak J, Chatterji B, Londhe KB, Watkins PB: Serum acute phase reactants hallmark healthy individuals at risk for acetaminophen induced liver injury. Genome Med 2013, 5:86.

2. Amacher DE, Schomaker SJ, Aubrecht J: Development of blood biomarkers for drug-induced liver injury: an evaluation of their potential for risk assessment and diagnostics. Mol Diagn Ther 2013. doi: 10.1007/ s40291-013-0049-0.

3. Schomaker S, Warner R, Bock J, Johnson K, Potter D, Van Winkle J, Aubrecht $\mathrm{J}$ : Assessment of emerging biomarkers of liver injury in human subjects. Toxicol Sci 2013, 132:276-283.

4. Antoine DJ, Dear JW, Lewis PS, Platt V, Coyle J, Masson M, Thanacoody RH, Gray AJ, Webb DJ, Moggs JG, Bateman DN, Goldring CE, Park BK: Mechanistic biomarkers provide early and sensitive detection of acetaminophen-induced acute liver injury at first presentation to hospital. Hepatology 2013, 58:777-787.

5. Antoine DJ, Jenkins RE, Dear JW, Williams DP, McGill MR, Sharpe MR, Craig DG, Simpson KJ, Jaeschke H, Park BK: Molecular forms of HMGB1 and keratin-18 as mechanistic biomarkers for mode of cell death and prognosis during clinical acetaminophen hepatotoxicity. J Hepatol 2012, 56:1070-1079.

6. Craig DG, Lee P, Pryde EA, Masterton GS, Hayes PC, Simpson KJ: Circulating apoptotic and necrotic cell death markers in patients with acute liver injury. Liver Int 2011, 31:1127-1136.

7. Dear JW, Antoine DJ, Starkey-Lewis P, Goldring CE, Park BK: Letter to the Editor: Early detection of paracetamol toxicity using circulating liver microRNA and markers of cell necrosis. Br J Clin Pharmacol 2013. doi: 10.1111/bcp.12214.

8. Daly AK, Donaldson PT, Bhatnagar P, Shen Y, Pe'er I, Floratos A, Daly MJ, Goldstein DB, John S, Nelson MR, Graham J, Park BK, Dillon JF, Bernal W, Cordell HJ, Pirmohamed M, Aithal GP, Day CP, DILIGEN Study; International SAE Consortium: HLA-B*5701 genotype is a major determinant of druginduced liver injury due to flucloxacillin. Nat Genet 2009, 41:816-819.

9. Lucena MI, Molokhia M, Shen Y, Urban TJ, Aithal GP, Andrade RJ, Day CP, Ruiz-Cabello F, Donaldson PT, Stephens C, Pirmohamed M, Romero-Gomez M, Navarro JM, Fontana RJ, Miller M, Groome M, Bondon-Guitton E, Conforti A, Stricker BH, Carvajal A, Ibanez L, Yue QY, Eichelbaum M, Floratos A, Pe'er I, Daly MJ, Goldstein DB, Dillon JF, Nelson MR, Watkins PB: Susceptibility to amoxicillin-clavulanate-induced liver injury is influenced by multiple HLA class I and II alleles. Gastroenterology 2011, 141:338-347.

10. Urban TJ, Shen Y, Stolz A, Chalasani N, Fontana RJ, Rochon J, Ge D, Shianna KV, Daly AK, Lucena MI, Nelson MR, Molokhia M, Aithal GP, Floratos A, Pe'er I, Serrano J, Bonkovsky H, Davern TJ, Lee WM, Navarro VJ, Talwalkar JA, Goldstein DB, Watkins PB, Drug-Induced Liver Injury Network; DILIGEN; EUDRAGENE; Spanish DILI Registry; International Serious Adverse Events Consortium: Limited contribution of common genetic variants to risk for liver injury due to a variety of drugs. Pharmacogenet Genomics 2012, 22:784-795.

doi:10.1186/gm489

Cite this article as: Aubrecht et al:: Emerging hepatotoxicity biomarkers and their potential to improve understanding and management of drug-induced liver injury. Genome Medicine 2013 5:85. 